\title{
Comparison Between Circulating Immune Complexes and Prostate Specific Antigen to Assess the Pathogenesis of Prostate Cancer
}

\author{
Alka Vishwas Nerurkar ${ }^{1}$, Desai Jyotsna ${ }^{1}$, Mukund Andankar ${ }^{2}$, Annada Joshi ${ }^{1}$, Sneha Shinde ${ }^{3}$ \\ ${ }^{1}$ Department of Biochemistry, T. N. Medical College \& B. Y. L. Nair Ch. Hospital, Mumbai, Maharashtra, India \\ ${ }^{2}$ Department of Urology, T. N. Medical College \& B. Y. L. Nair Ch. Hospital, Mumbai, Maharashtra, India \\ ${ }^{3}$ T. N. Medical College \& B. Y. L. Nair Ch. Hospital, Mumbai, Maharashtra, India
}

Email address:

alkanerurkar@yahoo.com (A. V. Nerurkar), annada.joshi@gmail.com (A. Joshi)

\section{To cite this article:}

Alka Vishwas Nerurkar, Desai Jyotsna, Mukund Andankar, Annada Joshi, Sneha Shinde. Comparison Between Circulating Immune Complexes and Prostate Specific Antigen to Assess the Pathogenesis of Prostate Cancer. Journal of Cancer Treatment and Research. Vol. 5, No. 3, 2017, pp. 43-47. doi: 10.11648/j.jctr.20170503.13

Received: February 20, 2017; Accepted: March 22, 2017; Published: April 12, 2017

\begin{abstract}
Prostrate cancer is a common cancer in males with increasing rate of incidence these days. Two known markers prostatic acid phosphatase (PAP) and prostate specific antigen (PSA) have been used for diagnosis and in the clinical management of prostate cancer patients. PAP and PSA tests are not specific and hence not reliable. Therefore, certain immunological tests need to be developed to aid the clinicians in early detection, metastasis and recurrence and also to follow clinical course of the disease and/or its response to therapy. The present study shows that the circulating immune complexes (CICs) may be associated with the pathogenesis of prostate cancer and their progression. CICs were investigated in 50 prostate cancer patients having different grades of the disease and 45 benign prostate hyperplasia patients (BPH). Estimation of CICs was done using 3.75\% Polyethylene glycol 6000 (PEG) serum precipitation. The results obtained were compared with normal healthy individuals. $83.33 \%$ and $91.66 \%$ positivity was observed in Grade I and Grade II prostate carcinomas respectively, whereas $100 \%$ positivity was observed in Grade III and Grade IV prostate carcinoma. BPH patients showed $60 \%$ positivity. Strong positive correlation $(\mathrm{r}=0.5748)$ between Prostate specific antigen (PSA) and CIC was observed in prostate cancer patients. Present investigation shows CICs may be associated with the pathogenesis of prostate cancers. The further qualitative analysis of CICs may help us to formulate specific immunological tests for early detection, management and monitoring the efficacy of treatment in prostate cancers.
\end{abstract}

Keywords: Circulating Immune Complexes (CICs), Benign Prostate Hyperplasia Patients (BPH), Prostate Specific Antigen (PSA), Prostate Cancer (PCa), Polyethylene Glycol 6000 (PEG)

\section{Introduction}

Prostate cancer is a major malignancy in males. It is the second most common cause of cancer death in western countries [1], [2]. Now days the incidence of prostate cancer is rising even in India. It currently ranks $2^{\text {nd }}$ in incidence and $4^{\text {th }}$ in cancer mortality for men in Mumbai [3]. Also, its incidence is increasing by $2.5 \%$ every year among the Indian races [4], [5].

Cancer of the prostate is a disease that is becoming increasingly common as the population ages; but in spite of intensive research our knowledge of the basic factors responsible for its initiation andgrowth are almost as limited now as they were 40 years ago. Without the basic knowledge it is impossible to develop a logical basis for prevention, diagnosis and treatment of any disease.

Two well-known markers, prostatic acid phosphatase (PAP) and prostate specific antigen (PSA) have been used for diagnosis and in the clinical management of prostate cancer patients. Prostate specific antigen (PSA) is more sensitive and reliable marker than prostatic acid phosphatase (PAP) [6], [7]. PSA is produced by secretory epithelial cells of the 
prostate gland and drains into the ductal system, where it catalyzes the liquefaction of the seminal coagulum after ejaculation. Its serum levels are normally less than $4 \mathrm{ng} / \mathrm{ml}$ but mayvary according to the patient's age and race; any process that disrupts the normal architecture of the prostate which allows diffusion of PSA into thestroma and microvasculature [8], [9]. Elevated serum PSA levels are seen with prostatitis, infarcts and hyperplasia, transiently after biopsy, but the most clinically important increase is seen with prostatic adenocarcinoma. Cancer actually produces less PSA per cell than benign epithelium, but the greater number of malignant cells and the stromal disruption associated with cancer account for the increased serum PSA levels [10], [11]. Though the PSA test is the first step towards a cancer diagnosis, it lacks specificity because elevated PSA levels may signal conditions other than cancer like $\mathrm{BPH}$ and prostatitis. Thus, PSA can no longer be considered as a classical tumor marker whose levels are directly correlated with increasing stage of the disease [12], [13], [14].

Basic immunology research over several decades has led to an improved understanding of tumor recognition by components of the immune system and mechanism of tumor evasion from immune detection [15], [16], [17]. Many investigators have attempted to develop biochemical and immunological tests for detection of and quantitation of such tumor markers [18]. The main aim of such studies on markers is to aid the cliniciansin early detection, metastasis and recurrence and also to follow clinical course of the disease and/or its response to therapy.

The level and characteristic of circulating immune complexes (CIC) present in sera of cancer patients have shown both good and poor correlation with the disease progress [19], [20], [21], [22]. As far as prognosis is concerned many authors have observed a variety of correlation between CIC levels and disease prognosis ranging from partial to very good [23], [24]. It has been demonstrated that the detection of antigen specific CICs in the sera of cancer patients may provide an approach for a more precise definition of the nature of the tumor antigen important for immune prognosis [25], [26], [27]. Detailed characterization of CICsshould enable both the detection and identification of their antigenic component [28]. Disease specific antigens have been identified by immune complex analysis in infectious diseases [29] and neoplastic disorders [30], [31]. Approach to such identification may enable us not only to develop specific immunological test for diagnosis and prognosis of prostate cancer patients but also prove to be useful as adjunct treatment modality in prostate cancer.

\section{Materials and Methods}

The present study included 50 prostate cancer patients (age range 40-75 years), 45 patients diagnosed as BPH (age range 40-75 years) and 25 normal subjects (age range 25-50 years) who were not having any major illness in the past. All the samples were obtained from the department of Urology, B. Y. L. Nair hospital and Tata memorial hospital, Mumbai. Informed consent form was taken from patients as per Ethic Committee rules. None of the patients had received any treatment prior to study. The data sheet of each patient was maintained inclusive of their economic status, dietary habits, age, sex, family history, their major past illness, habits such as consumption of alcohol, smoking, serum PSA levels, etc.

Polyethylene Glycol Mediated CIC Precipitation

Approximately $20 \mathrm{ml}$ of venous blood samples from the subjects were collected in sterile dry glass tubes and kept in incubator at $37^{\circ} \mathrm{C}$ for 3-4 hours. The serum was then aspirated, clarified by centrifugation at $1500 \mathrm{rpm}$ for $10 \mathrm{mins}$ and used for measurement of CIC levels using 3.75\% PEG 6000 in borate buffer [27]. One part of the freshly obtained serum was mixed with two parts of $0.01 \mathrm{M}$ borate buffer $\mathrm{pH}$ 8.4. To this mixture, 27 parts of $4.166 \%$ PEG was added (final serum dilution 1:30 and serum PEG concentration $3.75 \%$ ). After incubation at room temperature for $60 \mathrm{~min}$ the turbidity developed was measured at $450 \mathrm{~nm}$ using spectrophotometer against control containing 1:30 diluted serum in borate buffer without PEG. The levels of CIC in the serum were expressed in terms of OD $450 \mathrm{~nm}$ measured at the end of $60 \mathrm{~min}$.

\section{Results}

\subsection{CIC Levels in Sera of PCa, BPH and Normal Donors}

Table 1 represents the CIC levels in serum samples from 25 normal donors, 45 benign prostatic hyperplasia patients and 50 prostate cancer patients of different grades. The values in Table 1 show a significant and obvious difference in the categories studied. The mean levels of CIC in categories of patients were higher than that in the normal donors. Since all samples from normal donors showed some variable levels of CIC, the turbidity value at (mean+2SD) for normal samples i.e. 0.133 was used as cut off limit. All the samples giving turbidity value above this level were considered to be positive. The discriminating values put $4 \%$ of normal samples above the cut off limit and thereby render them positive. The frequency of positive serum samples was found to be very high in prostate cancer $(83.33 \%$ to $100 \%)$ whereas $60 \%$ of BPH patients were positive for CIC levels. The statistical evaluation of these results shows that the mean CIC levels in BPH and prostate cancer differ significantly from mean level in normal subjects $(p<0.001)$. The elevated levels of CIC were found in all different grades of prostate cancer. In the present study it was found that as the disease progresses from well differentiated (Grade I) to poorly differentiated (Grade IV) form, there is a significant increase in CIC levels $(\mathrm{p}<0.001)$. 
Table 1. Levels of CIC in normal subjects, BPH and different grades of prostate cancers.

\begin{tabular}{lllllll}
\hline \multicolumn{2}{l}{ Turbidity value at $\mathbf{\Delta} \mathbf{4 5 0} \mathbf{n m}$} & & & & & \\
\hline Subjects & No. of samples & Range & Mean & SD & SE & \% Positive samples \\
\hline Normals & 25 & $0.024-0.141$ & 0.0674 & 0.03278 & 0.00655 & $4 \%$ \\
BPH & 45 & $0.026-0.496$ & 0.1879 & 0.10910 & 0.01626 & $60 \%$ \\
Grade I & 12 & $0.116-0.326$ & 0.2247 & 0.06879 & 0.01986 & $83.33 \%$ \\
Grade II & 12 & $0.106-0.367$ & 0.2879 & 0.07541 & 0.02177 & $91.66 \%$ \\
Grade III & 8 & $0.325-0.401$ & 0.3643 & 0.02413 & 0.00853 & $100 \%$ \\
Grade IV & 18 & $0.265-0.497$ & 0.3936 & 0.06133 & 0.01445 & $100 \%$ \\
\hline
\end{tabular}

Grade I: w/d PCa- well differentiated prostate cancer (Gleason's score between 2-4)

Grade II: m/d PCa- moderately differentiated prostate cancer (Gleason's score between 5-6)

Grade III: pm/d PCa- poorly moderately differentiated prostate cancer (Gleason's score between 7-8)

Grade IV: p/d PCa- poorly differentiated prostate cancer (Gleason's score between 8-10)

\subsection{Comparison of CIC and PSA Levels in Sera of PCa and $\mathrm{BPH}$}

In the present investigation, the clinical data obtained for PSA in PCa and BPH was compared with the corresponding CIC levels. Table 2 indicates the mean PSA and CIC values in prostate cancer and BPH. On correlating the levels of CIC and PSA in PCa and BPH patients, a significant positive correlation $(\mathrm{p}<.001)$ can be observed with a correlation coefficient ( $\mathrm{r}$ ) of 0.5748 .

Table 2. Comparison of CIC levels and PSA levels in BPH and prostate cancer patients. A good correlation with correlation coefficient $(r)$ is 0.5748 which is found to be significant $(p<0.0001)$.

\begin{tabular}{llll}
\hline Parameter & & BPH & Prostate Cancer \\
\hline \multirow{2}{*}{ CIC } & $\mathrm{N}$ & 45 & 50 \\
& $\mathrm{X} \pm \mathrm{SE}$ & 0.20419 & 0.33610 \\
\multirow{2}{*}{ PSA } & $\mathrm{N}$ & 45 & 50 \\
& $\mathrm{X} \pm \mathrm{SE}$ & 14.6667 & 484.777 \\
\hline
\end{tabular}

\section{Discussion}

The occurrence of circulating immune complexes in the sera of patients with various types of neoplasia is now well established [32], [33]. This is also evident in prostate cancer [21]. Over the last decade several attempts have been made to determine the usefulness of elevated serum levels of CIC as marker for tumor burden and prognosis [24].

Using simple technique of PEG mediated precipitation of CIC, the present investigation has revealed that the CIC levels in sera of $\mathrm{PCa}$ and $\mathrm{BPH}$ are significantly elevated in comparison with the levels of normal subjects $(\mathrm{p}<.001)$. It is also observed in $\mathrm{PCa}$ that as the disease progresses from well differentiated (Grade I) to poorly differentiated (Grade IV) form, there is a significant increase in CIC levels $(p<.001)$. These results are in consistent with those obtained by other workers [21], [32], [33]. The increased levels of CIC obtained with the grades of disease suggest that the CIC is likely to contribute in evaluating the degree of malignancy and it may be associated with poor prognosis. Kobayashi K. et al also indicated the relationship between the levels of CIC with tumor size, invasive propensity and virulence levels in bladder and other urogenital cancers [36].

Golda R et al [24] who have indicated the elevated levels of
CIC in $66.7 \%$ of patients with adenocarcinoma of prostate and $38 \%$ of those with BPH whereas Sakai et al found considerably lower percentage of positivity (18.8\%) in prostate carcinoma.

Many researchers [34], [35], [37], [38] reported that the antigenic part of CICs originate from the diseased tissues. Release of antigen from these tissues increases as the disease progresses and that may be responsible for increase in the levels of CICs with increasing grades of $\mathrm{PCa}$ which is observed in the present study. The above findings may help in predicting the malignant transformation of premalignant lesion like BPH but follow up study with large number of samples is required to draw any further conclusion.

Presently PSA is the only diagnostic marker available for prostate cancer evaluation. But it lacks the specificity as its levels are elevated in many other benign conditions. In present study we observed strong positive correlation of PSA with CIC which can have the diagnostic value. In future it would be of great interest to examine the protein fingerprint of CIC samples to detect associated qualitative changes in CIC composition which will help us to formulate specific immunological test. Such study is in progress.

\section{Conclusion}

From the present investigations it is evident that the levels of CICs differ significantly from normal subjects to BPH patients and prostate cancer patients. It is also evident that elevated levels are observed in different grades of prostate cancer. As the disease progresses from Grade I to Grade IV, the levels of CICs also increase ( $<<0.001)$.

When clinical data obtained for PSA in prostate cancer patients and BPH patients was compared with the corresponding CIC levels, the results clearly show a significant positive correlation with a correlation coefficient ( $\mathrm{r}$ ) of 0.5748 .

The results indicate that if the qualitative analysis of CIC is done then there is possibility of developing a specific immunological test that may help for early diagnosis, prognosis, evaluation of efficacy of treatment and recurrence in prostate cancer patients.

\section{Acknowledgement}

The authors are thankful to Research Society, T. N. 
Medical College and B. Y. L. Nair hospital for providing financial support for the project.

\section{References}

[1] American Cancer Society, Cancer Facts and Figures, 2008, Atlanta.

[2] Olivier Cussenot, Genetic susceptibility to prostate cancer. The Prostate-New concepts and Developments, Special Pharma Edition 2006, Edited by- Fouada K Habib, Taylor and Francis Group, London and New York, p 49-56.

[3] Jain S, Saxena S, Kumar A. Epidemiology of prostate cancer in India. Meta Gene 2014 Dec; 2: 596-605.

[4] Yeole BB, Jussawala DJ. Descriptive epidemiology of the cancers of male genital organs in greater Bombay. Indian $\mathrm{J}$ cancer 1997; 34: 30-35.

[5] Sexually active elderly less prone to prostate cancer. Tribune News Service, Monday, February 28, 2005, Chandigarh, India.

[6] Chu TM. Prostate cancer- associated markers. Immunol. Ser. 1990; 53: 339-356.

[7] Van Dalen, Helmount DH, Van Caugergh RD. The contribution of prostatic acid phosphatase and prostate specific antigen in the diagnosis of prostatic cancer. Int. J. Bio Markers, 1998 Apr-Jun; 3 (2): 123-126.

[8] Polascik TJ, Oesterling JE, and Partin AW."Prostate Specific Antigen: A Decade of Discovery - What we have learned and where we are going". J Urol. 1999; 162 (2): 293-306 (review).

[9] Thompson IM, Pauler DK, Goodman PJ, et al. Prevalence of prostate cancer among men with a prostate specific antigen level< or=4.0 ng per milliliter. New England Journal of Medicine 2004; 350 (22): 2239-2246.

[10] Bostwick DG. Prostate specific antigen - Current role in diagnostic authology of prostate cancer. Am. J. ClinPathol. 1994 Oct; 102 (4 suppl 1): S31-7.

[11] Leibovici D, Spiess PE, Agarwal PK, Tu SM, Pettawat CA, Hitzhusen K, Millikan RE, Pisters LL. Prostate cancer progression in the presence of undetectable or low serum prostate-specific antigen level. Cancer. 2007 Jan 15; 109 (2): 198-204.

[12] Tenke p, Horti J, Balint P, Kovacs B. Prostate cancer screening. Recent Results. Cancer Res. 2007; 175: 65-68.

[13] Keetch DW, Catalona WJ, Smith DS. Serial prostatic biopsies in men with persistently elevated serum prostate specific antigen Values. J Urology 1994; 151 (6): 1571-1574.

[14] Benson MC, Whang GS Olsson CA. The use of prostate specific antigens density to enhance the predictive value of intermediate Levels of serum prostate specific antigens. J Urology 1992 Mar; 147 (3pt 2); 817-821.

[15] McNeel DG. Prostate cancer immunotherapy. Curr. OpinUrol, 2007 May: 17 (3): 175-181.

[16] Kipp RT, McNeel DG. Immunotherapy for Prostate cancerrecent progress in clinical trials. ClinAdv Hematology Oncol. 2007 Jun; 5 (6): 465-474, 477-479.
[17] Elkord E. Immunology and Immunotherapy approaches for prostate cancer. Prostate Cancer Prostatic Dis. 2007; 10 (3): 224-236.

[18] Stenman UH, Abrahamsson PA, Aus G, Lilja H, Bangma C, Hamdy FC, Boccon-Gobod L, Ekman P. Prognostic value of serum markers for prostate cancer. Scand J UrolNephrol Suppl. 2005 May; (216): 64-81.

[19] Sakai S, Kato N, Ito Y et al. Detection of circulating immune complexes by polyethylene glycolprecipitation complement Consumption test in urological malignant disease. Hinyokikokiy, 1984: 30; 1611-1617.

[20] Asis M, Akhtar S, Malik A. Evaluation of cell mediated and circulating immune complexes as prognostic indicators in cancer patients. Cancer detection Prev, 1998; 22 (2): 87-99.

[21] Dass TK, Aziz M, Rattan A. Prognostic significance of circulating C patients. Jap J. Cancer Res, 1991 Nov; 82 (11); 1284-1294.

[22] Bhatti R, Shaw M, Ray P. Inhibition of sensitized leucocyte's in vitro reactivity by circulating complexes in prostate cancer. Cellular and Molecular Life Sciences 1987 Aug 15; 43 (8): 916-917.

[23] Theofilopoulos AN. Immune complexes in cancer. N. Eng J. Med 1982; 307: 1208-1209.

[24] Golda R, Wolski Z, Wyszomirska, Golda M, Madalainskik, Michlkiewicz J. The presence and structure of circulating immune complexes in patients with prostate tumors. Med SciMonit, 2004; 10 (3): CR 123-127.

[25] Ziccardi RJ. The role of immune complexes in the activation of the first component of human complement. J. of Immunol. 1984 Jan; 132 (1): 283-288.

[26] Philips TM, Queen WD and Lewis MG. The significance of circulating immune complexes in patients with malignant melanoma, in: Melanoma Antigen and Antibodies. (RA. Reisfeld and S. Ferrone, eds.), 1982; pp. 289-316.

[27] Salinas FA, Wee KH and Silver HKB. Immune complexes and human neoplasia: Detection and quantitation of circulating immune complexes by the fetal liver cell assay. Cancer Immunology, Immunother. 1981b; 12: 11.

[28] Chu TM, Kuriyama M, Johnson E, Papsidero LD, Killian CS, Murphy GP, Wang MC. Circulating antibody to prostate antigen in patients with prostatic cancer. Ann N Y Acad Sci. 1983; 417: 383-389.

[29] Almedia JD and Waterson AP. Immune complexes in hepatitis. Lancet II 1969; 983-986.

[30] Nerurkar A V, Advani S H, Gothaskar B P. Circulating immune complexes in Hodgkin's disease. Neoplasm 1989; 36 (2): 199-205.

[31] Long J C, Hall C L, Brown C A, Stamalos et al. Binding of soluble immune complexes in serum of patients with Hodgkin's disease to tissue culture derived from the tumor. New Eng J of Medicine 1977; 297: 295- 99.

[32] Rhia I, Haskova V, Kaslik J, Maierova M, Stransky J. The use of polyethylene glycol for immune complexes detection in human sera, Molecular Immunology 1977; 16: 489-93.

[33] Laemmli U K. Nature 1970; 227: 680- 89. 
[34] Cynthia Jane, Nerurkar A V, Karjodkar F R. Circulating immune complexes marker for disease progress in oral cancer. Indian J of Clinical Biochemistry 2007; 22 (2): 114-17.

[35] Nerurkar A V, Advani S H, Gothaskar B P. Circulating immune complexes in Hodgkin's disease. Reactivity of IgG isolated from circulating immune complexes. Neoplasm 1993; 40 (2): 87-92.

[36] Kobayashi k, Umeda T, Akaza H E, et al. Circulating immune complexes in cancer patients with bladder cancer patients with bladder cancer and malignancies of the urogenital tract. Urol Int. 1984; 39: 232-35.
[37] Beneduce L, Prayer Galetti T, Giustinian A M, Gallota A, Betto G, Pagano F, Fassina G. Detection of prostate specific antigen coupled to immunoglobulin $\mathrm{M}$ in prostate cancer patients. Cancer Detect Prev. 2007; 31 (5): 402-07.

[38] Wiederkehr F, Bueler M R, Vondersehmitt D J. Chromatographic and electrophoretic studies of circulating immune complexes in plasma. J Chromatography 1991; 566: 77-78. 